\title{
Video Distortion Removal in Effect of Non-Linear Patch Model
}

\author{
Er. Manisha Sharma ${ }^{1}$ and Er. Kiran Gupta ${ }^{2}$ \\ Department of Computer Science Engineering \\ Swami Devi Dyal Institute of Engineering and Technology \\ Golpura, Barwala, Panchkula, India \\ ${ }^{1}$ mishi.720@gmail.com, ${ }^{2}$ er.kirangupta@gmail.com
}

\begin{abstract}
Video generation is the strategy of making videos by discovering moving pictures (videography) and making blends in live creation. During this aspect of videos creation, sequence of images gets contorted in the image acquisition and transmission phase. This paper presents a non-linear patch model for enhancing the degraded quality of video sequences. The proposed method navigates the frames of videos by selecting a patch of considerable width and with the assistance of this patch, a regression model is applied in order to have a robust effect while performing de-noising, de-blurring or superresolution. The proposed work implements on three models i.e. search model, regression model and non-linear patch model. Various filters have been propounded e.g. kernel filters, total variation filters, adaptive median filter etc. Regression model is applied to every frame with predefined number of iterations with estimated number of frames. The proposed method inculpates two kinds of noise i.e. Gaussian noise and impulse noise. Various performance comparison metrics have been evaluated to check the coherence and productivity of imaging system like Peak signal to noise ratio (PSNR), Mean squared error (MSE), Root mean squared error (RMSE), Standard deviation (SD), Linear correlation and structural entropy.
\end{abstract}

Keywords: MSE, non-linear patch model, PSNR, Restoration, SD

\section{Introduction}

Image processing is a methodology to perform out a couple of operations on an image, remembering the deciding objective to get an enhanced picture or to concentrate some huge data from it. The innovation of picture preparing is worried with a control of the components of a photo to improve its data content. Advanced picture handling incorporates the usage of digital computer for the required operations [5]. From early period to current time pictures have been utilized to make sense of or to remain for something. With the appearance of present day century recordings came into way of life, there are various applications where high esteem picture and video has its significance like cosmic imaging, attractive reverberation imaging, visual discernment, engineering, military applications and so on.

A picture is a continuous two dimensional conveyance of luminance or some other visible impact and video is connection of pictures to shape a worldview and the process of evacuating commotions in the pictures while attempting to save however much as elements as could be expected is termed as restoration. The motivation behind restoration is to "adjust for" or "fix" deformities which debase an image. This type of degradation occurs during acquisition and transmission phase of image processing. The degradation can be of any type like occurring blur, motion mis-focus, faulty communication channel etc., [1]. Two types of noises are inserted to check the restoration process i.e. Gaussian noise and impulse noise. At the point when the debasement of an image is brought about as a result of the presence of noise, the most ideal method for recouping the picture is to 
compensate for the cause that has created corruption i.e., expulsion of the commotion. The problem formulation aims to have a restoration system which can establish the video caught by low end gadgets like mobile with fluctuating resolutions and under unconstrained light and environment. To the extent the quality is concerned, the nature of the decoded picture is corresponding to the measure of received information. So an essential picture is fabricated and its quality is increased progressively and also it is accomplished for the determination.

In all image processing systems, image restoration plays a major role and it forms the major part of image processing systems. The vital concern is to refine an image or sequence of images from its contorted stage and this can be achieved by fulfilling the objective requirements i.e., to preserve the structural detail while restoring and by improving the correlation linearity between video frames.

The remainder of this paper is organized as follows: Section 2 describes the contribution of various authors to perform considerable measure of work in the field of image and video restoration. The entire methodology has been presented in the Section 3 together with flowchart of the proposed model. Evaluation parameters such as PSNR, correlation, MSE, Entropy, SD are explained in Section 4. Section 5 completes with the results that have been formulated with the help of proposed method and various comparison metrics are also defined. The whole paper is ended up in the section VI that states the conclusion and future scope of the proposed topic.

\section{Related Work}

Several methods have been proposed in the direction of image and video restoration by different researchers via Wavelet transform, isotropic and anisotropic filtering, blind deconvolution, wiener filtering, augmented lagrangain method [13, 8, 7, 1, 9]. There are other advanced methods as well and the main motive is to remove distortion from the degraded image sequences. Malfait in [13] proposed the method of wavelet transform for the concealment of noise in pictures. The strategy depends on two measures. The first is a great measure of smoothness of the picture and depends on the type of the wavelet coefficients. The second, novel measure considers geometrical imperatives, which are by and large legitimate for common pictures. Another author Harrabi in [8] proposed a technique of regular anisotropic diffusion. The regularized anisotropic diffusion method i.e., RADM strategy was utilized for showing critical improvement in execution contrasted with isotropic and anisotropic techniques, utilized for separating the same pictures, which will be then fragmented by calculation fuzzy c-means (FCM). Patil in [7], presented Bayes spectral entropy with the help of Discrete Cosine Transform (DCT) for doing image restoration by identifying blur estimation. DCT method has been widely used because its computation time and cost is very low as compared to other wavelet transform techniques. DCT has an additional property of high energy compaction that makes it suitable for blur estimation. An approach of adaptive wiener channel design was presented by Trambadia in [1] to establish again the debased picture. This method proposed wiener channel with gradient based smoothing and median channel with an adaption of bilateral channel for edge saving and guided channel for diminishing staircase impact and angle inversion. Mairal, J., in [11] proposed to bring together two distinctive ways to deal with picture rebuilding: On the one hand, taking in a premise set adjusted to inadequate signal descriptions that has turned out to be extremely successful in picture recreation and classification errands. Then again, expressly misusing the self-likenesses of common pictures that has prompted the effective non-neighborhood which implies way to deal with picture reclamation. Maik, $\mathrm{V}$ in [3] proposed a method of adaptive video restoration which was used to abandon spatially varying blur in addition with the help of TCLS filter i.e., truncated constrained least squared channel. Nikolova in [10] depicted nonconvex non smooth regularization method for restoration and reconstruction. The 
arrangement of the nonconvex non smooth minimization issue is made out of steady areas encompassed by closed shapes and perfect edges. The primary objective was to grow quick minimization calculations to take care of the nonconvex non smooth minimization issue and concentrate on time optimization. Zhang, in [4] explained another HSI reclamation technique taking into account low-rank matrix recovery (LRMR), which can all the while expel the Gaussian commotion, impulse noise, deadlines, and stripes.

The main purpose had been to evacuate the noise level and to bring out the image or sequence of images to a level close to original one.

\section{Proposed Work}

The demand for image and video restoration has been increased extensively from past years. So the model that has been designed is to keep in mind the high value quality of images and videos. The proposed work is based on non-linear patch model. This model traverses the frames of videos by selecting a patch of some width and with the help of this patch, a regression model is applied in order to have a robust effect while performing denoising. The proposed work implements on three models i.e. search model, regression model and non-linear patch model. Various filters have been proposed in order to make restoration process an effective and robust one e.g. kernel filters, total variation filters, adaptive median filter etc. Regression model is applied to every frame with predefined number of iterations with estimated number of frames. The present work estimates that the elapsed time between frames needs to be minimized. The proposed method focuses on increasing the PSNR value and decreasing the MSE values to as much extent as possible. The randomness that occurs while distorting the image or sequence of images has to be improved and should be enhanced so that the transition from one frame to other becomes less complex and more efficient. The distortion has been applied in order to show a perspective view that under what conditions the need of restoration comes to play.

Following restoration filters are applied in the proposed system:

\section{A. Adaptive Median Filter}

Adaptive filters are fit for de-noising non-stationary pictures, that is, pictures that have sudden changes in power. Such channels are known for their capacity in naturally following an obscure situation or when a signal is variable with little from the earlier learning about the sign to be handled [6].

B. Total Variation Filter

A variation strategy which mutually handles movement estimation, moving article location, and movement obscure deconvolution. The proposed structure demonstrates the movement of obscure procedure and in addition shape and picture regularization terms, and comprehended by means of efficient regularized systems [12].

\section{Kernel Regression}

Regression examination is a measurable procedure for assessing the connections among variables. Kernel regression is a non-parametric strategy in measurements to evaluate the contingent desire of an arbitrary variable. The goal is to discover a non-direct connection between a couple of arbitrary variables $\mathrm{X}$ and $\mathrm{Y}$. Portion relapse is an estimation system to fit information. 


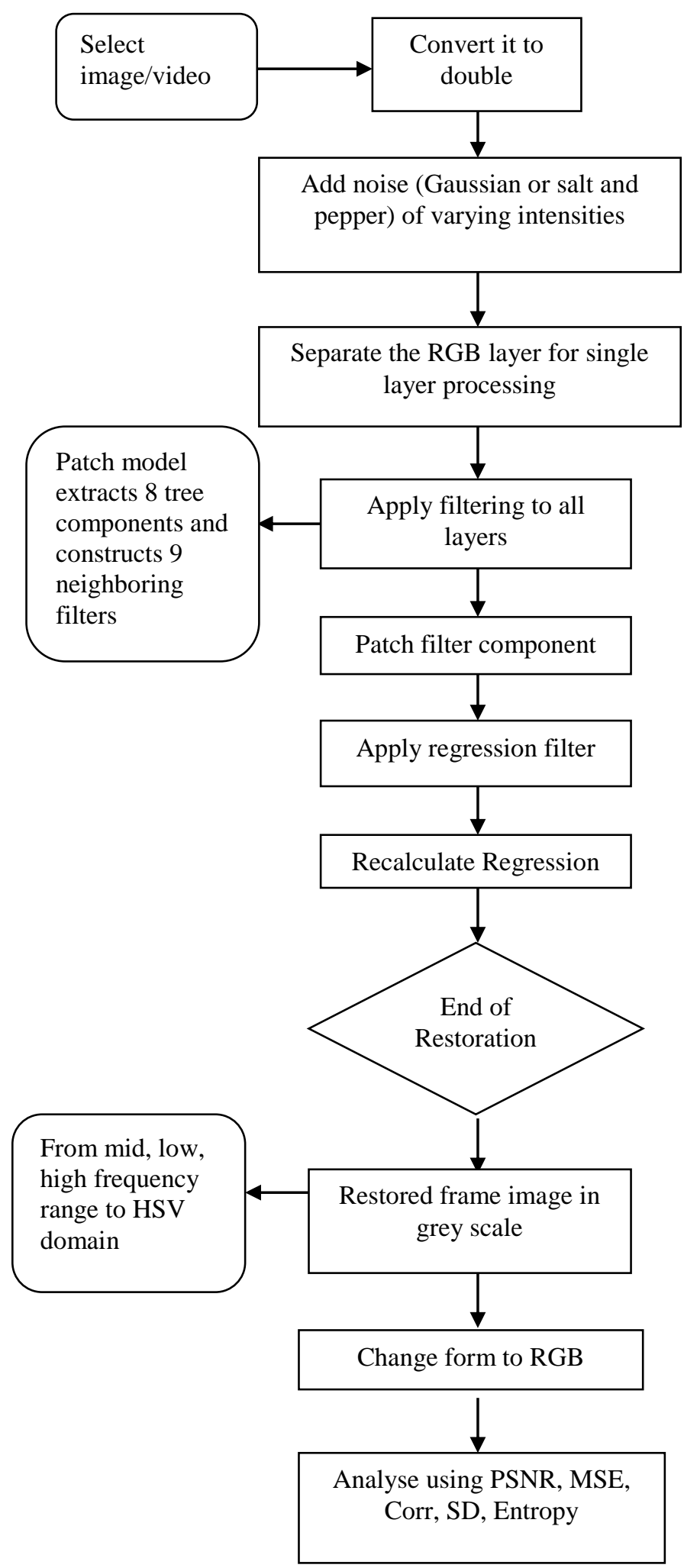

Figure 1. Block Diagram of Proposed Work

Figure 1 depicts the block diagram that gives the whole sequence of instructions that are required for the proposed system. Image or sequence of images i.e., video has been taken as an input and after adding distortion in it, the restored video will be yield as an 
output which will then be compared with various performance metrics like PSNE, MSE, RMSE etc.

Following are the steps that are required in designing the proposed system

Step1: Input the image or video which is to be restored.

Step2: Divide the video into its perspective frame data and separate the RGB layer for single layer processing.

Step3: Use the linear variation with the help of total variation filter to the selected block in the frame.

Step4: Process each frame block by block.

Step5: Apply adaptive median filter to the total number of blocks and calculate the median for the total number of blocks.

Step6: Now relate the mean from all blocks for calculating the total local variation among the individual blocks.

Step7: Apply regression model to each block of frame.

Step8: Now use these total variation values to design a selective size patch filter.

Step9: Now the calculated patch filter will be convoluted to proportionately reduce the noise values in the particular patch based on the local intensity, of the total variation mean calculated.

Step10: Change the form to RGB and analyze using various performance metrics i.e. PSNR, MSE, RMSE, Entropy, Correlation and Standard Deviation.

\section{Evaluation Parameters}

Following are the evaluation metrics that are used for evaluating the proposed work:

Peak signal to noise ratio (PSNR) - PSNR is the peak signal to noise ratio in decibels which is logarithmic scale. The PSNR is significant for information encoded regarding bits per sample, or bits per pixel. For instance, a picture with 8 bits for each pixel contains numbers from 0 to 255 [2]. The PSNR of the signal can be demonstrated by the equation.

$$
\text { PSNR }=10 \log \frac{(255)^{2}}{M S E}
$$

Here, the pixel is depicted by 8 bits per sample and MSE stands for mean squared error.

Mean squared error (MSE) - The mean squared error (MSE) is the squared standard of the distinction between the information and the estimate isolated by the quantity of components [2]. Mean Squared Error can be evaluated in one of numerous approaches to measure the distinction between qualities inferred by an assessment and the genuine quality being certificated. MSE is a danger capacity relating to the normal estimation of squared mistake.

MSE can be evaluated as:

$$
M S E=\frac{1}{M N} \sum_{j=1}^{M} \sum_{k=1}^{N}\left(X_{j, k}-X_{j, k}^{t}\right)^{2}
$$

Here, $x_{j, k}$ is the original image and $x_{j, k}^{v_{j}}$ is the restored image. 
Entropy - Entropy in the imaging system determines the relative randomness in the system which leads to violation of the imaging system. Entropy factor has to be reduced in all possible way to have an enhanced pattern [7].

$$
\eta=H(s)=\sum_{\mathrm{i}=1}^{n} p_{\mathrm{i}} \log _{2} \frac{1}{p_{\mathrm{i}}}=-\sum_{\mathrm{i}=1}^{n} p_{\mathrm{i}} \log _{2} p_{\mathrm{i}}
$$

Where $\mathrm{p}_{\mathrm{i}}$ is entropic probability, $\mathrm{N}$ is the no. of pixels,

$1 / \mathrm{p}_{\mathrm{i}}$ is the inverse probability, $\eta$ is the entropy of image.

Standard deviation - The standard deviation is a measure that is utilized to evaluate the measure of variety or scattering of an arrangement of information values. A low standard deviation shows that the information directs incline toward be near the mean (additionally called the normal worth) of the set, while an exclusive requirement deviation demonstrates that the information focuses are spread out over a more extensive scope of qualities.

Standard deviation of $(\mathrm{x} 1, \mathrm{x} 2, \ldots, \mathrm{xn})$ is

$$
S D=\sqrt{\frac{1}{N-1}\left(\sum_{i=1}^{N}\left(x_{i}-\bar{x}\right)^{2}\right)}
$$

$\mathrm{Xi}$ is the new image data, $\mathrm{X}$ is the Original image data and $\mathrm{n}$ is the total number of pixels.

\section{Results}

This section presents the whole proposed work results that have been formulated thoroughly in MATLAB. All results and various evaluation metrics are compiled and interpreted several times to check the compatibility of the proposed work.
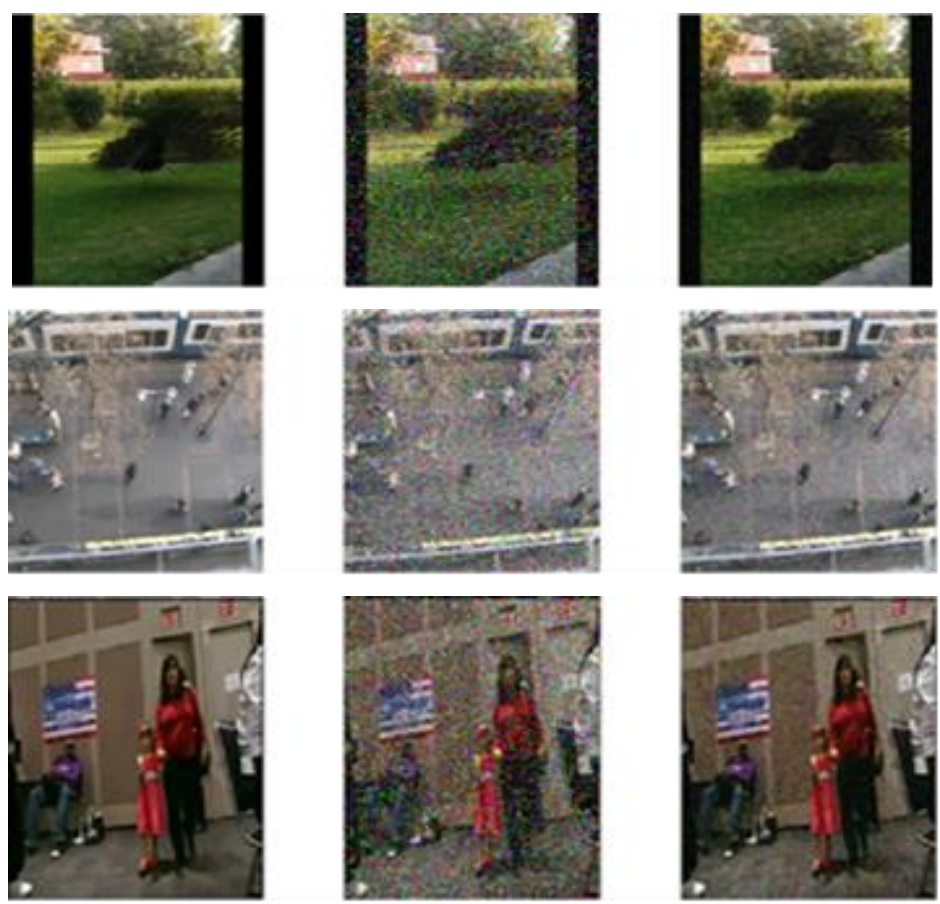

Figure 2. a) Original Video b) Corrupted by Gaussian and Impulse Noise c) Restored using Non-Linear Patch Model 
The Figure 2 shows the various videos that have been taken to see the efficiency of proposed method. The method is not confined to just one video. It can have various sets of resolutions of videos captured by low end gadgets that is the formulated problem. The first column in Figure 2 is the original video frame that is not distorted and in the middle confined a video frame corrupted by commotions i.e. Gaussian and impulse noise and third column yields the restored video frame that has better quality than contorted frame and close to original one.

Table 1. Evaluation Metrics for Restored Video of Peacock using Non-Linear Patch Model Corrupted by Gaussian Noise

\begin{tabular}{|c|c|c|c|c|c|}
\hline $\begin{array}{c}\text { No. of } \\
\text { Frames }\end{array}$ & PSNR & MSE & Entropy & $\begin{array}{c}\text { Standard } \\
\text { Deviation }\end{array}$ & Correlation \\
\hline 5 & 25.1713 & 0.0030 & 7.4582 & 33.6081 & 0.9797 \\
\hline 50 & 25.4012 & 0.0030 & 7.2583 & 33.9299 & 0.9740 \\
\hline 100 & 25.2600 & 0.0030 & 7.4349 & 34.9817 & 0.9809 \\
\hline
\end{tabular}

Table 2. Evaluation Metrics for Restored Video of Peacock using Non-Linear Patch Model Corrupted by Impulse Noise

\begin{tabular}{|c|c|c|c|c|c|}
\hline $\begin{array}{c}\text { No. of } \\
\text { Frames }\end{array}$ & PSNR & MSE & Entropy & $\begin{array}{c}\text { Standard } \\
\text { Deviation }\end{array}$ & Correlation \\
\hline 5 & 23.1386 & 0.0031 & 7.6450 & 33.5663 & 0.9995 \\
\hline 50 & 23.4809 & 0.0031 & 7.5211 & 34.3659 & 0.9817 \\
\hline 100 & 22.4802 & 0.0031 & 7.7728 & 35.0644 & 0.9943 \\
\hline
\end{tabular}

Table 1 describes the comparison of evaluation parameters corrupted by Gaussian noise and Table 2 explains with impulse noise. It is evident from the above two tables that as the number of frames and patch size increases, results are more accurate and efficiency is not violated.

Table 1 and 2 portrays various metrics that determines that peak signal to noise ratio between both the tables is different i.e. Gaussian noise is removed efficiently than impulse noise and quality is restored. Other facts that are entropy, standard deviation and correlation have also been increased in impulse noise. With the changes in the commotion the results vary to an extent. The proposed model works and behaves differently with variation in commotion.

Various Bar charts:

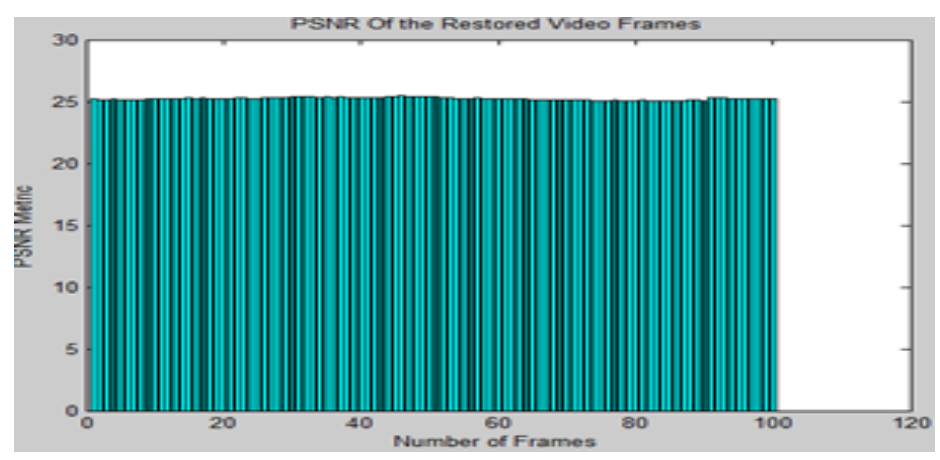

Figure 3. PSNR of Restored Frames 
Figure 3 presents the bar chart of PSNR value of restored frames with varying number of frames corresponding to Table 1. As the frames increases PSNR values show slight variation in them that determines the signal strength.

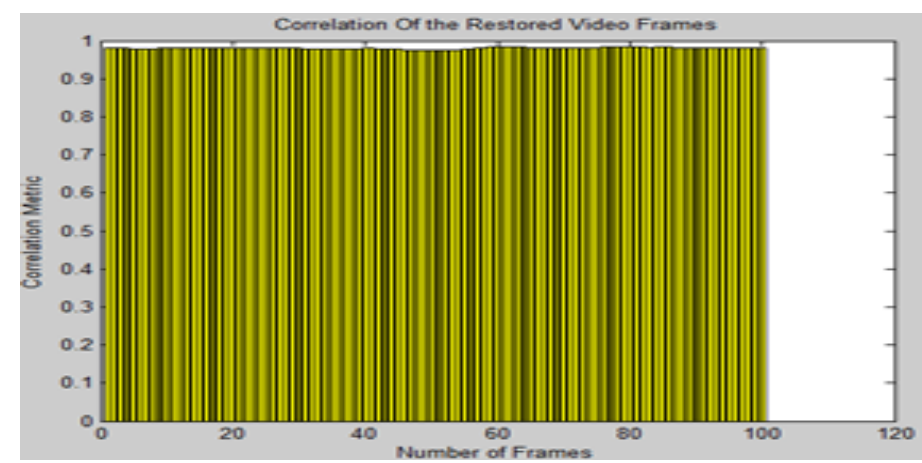

Figure 4. Correlation of Restored Frames

The bar chart of correlation of restored frames that were corrupted by Gaussian noise is depicted in Figure 4. The correlation parameter determines the linearity that one frame has with other frame. The correlation lies between 0-1 which shows the high quality of restored frames where 0 indicates poor quality and 1 indicates the high value.

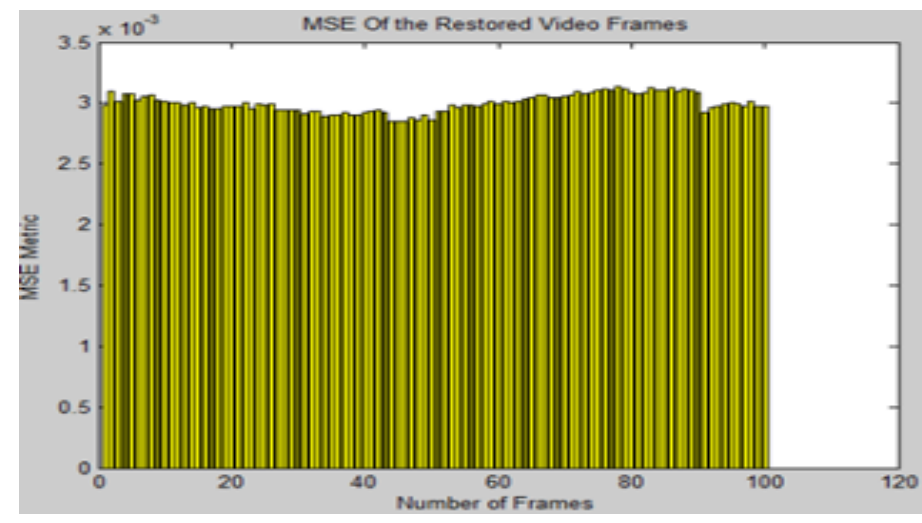

Figure 5. MSE of Restored Frames

Figure 5 shows the bar chart of major metric that governs the ratio of actual and estimated error i.e., MSE. As the PSNR value increases, MSE decreases that determines that there is low error rate in the restored frame. The MSE metric is stabled so as to have a clear estimate of actual error.

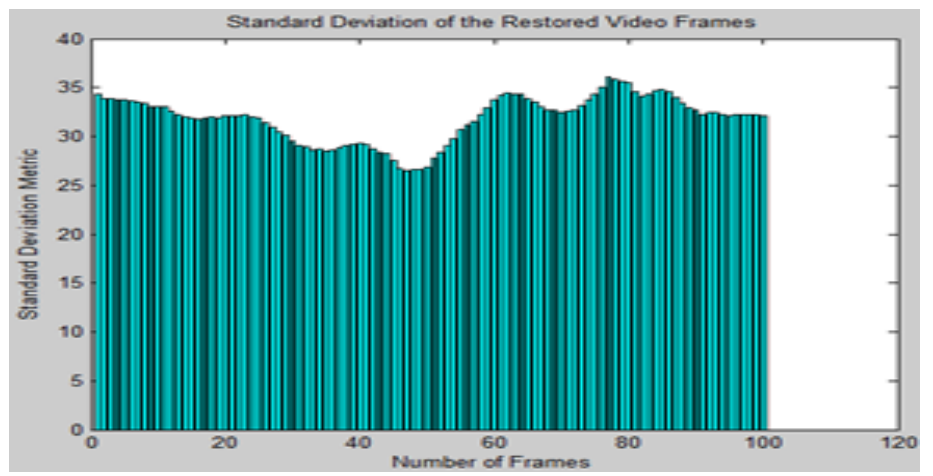

Figure 6. Standard Deviation of Restored Frames 
Standard Deviation depicts the variation that a restored frame exhibits and this is shown in Figure 6 with the help of bar chart of different number of frames.

Figures 3-6 shows evaluation metrics on $\mathrm{Y}$-axis and number of frames on $\mathrm{X}$-axis. These bar charts depicts various evaluation parameters under different number of frames and presents the variation that occurs when there is an increase in the frames and patch size of commotion.

\section{Conclusion}

Picture rebuilding is an essential branch of picture handling, managing the recreation of pictures by expelling noise and obscure from debased pictures and making them reasonable for human recognition. In this paper, the proposed non-linear patch model has been determined to complete the goal of restoration by applying the whole methodology on different colored videos and their performances have been compared with the evaluation metrics i.e., PSNR, MSE, Entropy, Correlation and Standard Deviation. The proposed non-linear patch framework has enhanced the rebuilding limit of images and videos in the current subjected environment. The application of the proposed system has been formulated in MATLAB using various videos. The experimental results presents that the peak signal to noise ratio value increases as the mean squared error decreases in the frames that are corrupted by two added noises i.e., Gaussian and impulse noise. PSNR depicts the high value of restored frames and MSE determines the error rate and proficiency. Randomness in the frames shows the variation of pixels, so entropy and SD has to be estimated to preserve the structural detail of restored frames. In future, digital imaging will become more compact and require less power consumption strategy. So, this technique can be enhanced by redundant discrete wavelet transform but that can take much memory space. RDWT can be used for high and commercial purposes.

\section{References}

[1] S. Trambadia and P. Dholakia, "Design and analysis of an image restoration using wiener filter with a quality based hybrid algorithms", In Electronics and Communication Systems (ICECS), 2015 2nd International Conference on IEEE, (2015) February, pp. 1318-1323.

[2] B. V. Bhalerao and R. R. Manza, "Rural Indian fingerprint image de-noising and techninques to remove noise for image enhancement and improve the recognition rate", In Computer and Communication Technology (ICCCT), 2014 International Conference on IEEE, (2014) September, pp. 67-72.

[3] V. Maik, H. Cheong, E. Chae, E. Lee, G. Jo, J. Paik and C. Park, "Spatially adaptive video restoration using truncated constrained least-squared filter", In Consumer Electronics (ISCE 2014), The 18th IEEE International Symposium on IEEE, (2014) June, pp. 1-2.

[4] H. Zhang, W. He, L. Zhang, H. Shen and Q. Yuan, "Hyperspectral image restoration using low-rank matrix recovery", Geoscience and Remote Sensing, IEEE Transactions, vol. 52, no. 8, (2014), pp. 47294743.

[5] W. Dong, L. Zhang, G. Shi and X. Li, "Nonlocally centralized sparse representation for image restoration", Image Processing, IEEE Transactions, vol. 22, no. 4, (2013), pp. 1620-1630.

[6] A. Nath, "Image Denoising Algorithms: A Comparative study of Different Filtration approaches used in image restoration", In Communication Systems and Network Technologies (CSNT), 2013 International Conference on IEEE, (2013) April, pp. 157-163.

[7] S. S. Patil, P. M. Kanjalkar and J. V. Kulkarni, "Blur estimation using polynomial curve fitting algorithm for image restoration using blind deconvolution", In Computing, Communications and Networking Technologies (ICCCNT), 2013 Fourth International Conference on IEEE, (2013) July, pp. $1-5$.

[8] R. Harrabi and E. Ben Braiek, "Isotropic and anisotropic filtering techniques for image denoising: A comparative study with classification”, In Electrotechnical Conference (MELECON), 2012 16th IEEE Mediterranean IEEE, (2012) March, pp. 370-374.

[9] S. H. Chan, R. Khoshabeh, K. B. Gibson, P. E. Gill and T. Q. Nguyen, "An augmented Lagrangian method for total variation video restoration", Image Processing, IEEE Transactions, vol. 20, no. 11, (2011), pp. 3097-3111.

[10] M. Nikolova, M. K. Ng and C. P. Tam, "Fast nonconvex nonsmooth minimization methods for image restoration and reconstruction", Image Processing, IEEE Transactions, vol. 19, no. 12, (2010), pp. 30733088 . 
[11] J. Mairal, F. Bach, J. Ponce, G. Sapiro and A. Zisserman, "Non-local sparse models for image restoration", In Computer Vision, 2009 IEEE 12th International Conference on IEEE, (2009) September, pp. 2272-2279.

[12] L. Bar, B. Berkels, M. Rumpf and G. Sapiro, "A variational framework for simultaneous motion estimation and restoration of motion-blurred video", In Computer Vision, 2007. ICCV 2007. IEEE 11th International Conference on IEEE, (2007) October, pp. 1-8.

[13] M. Malfait and D. Roose, "Wavelet-based image denoising using a Markov random field a priori model", Image Processing, IEEE Transactions, vol. 6, no. 4, pp. 549-565.

\section{Authors}

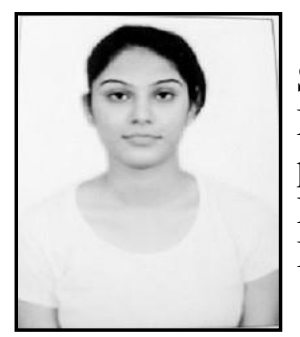

Er. Manisha Sharma is from Nangal. Born on 7 February 1993. She completed B. tech (Computer Science and Engineering) from Maharishi Markandeshwar University, Mullana, India in 2014. She is pursuing M. tech (Computer Science and Engineering) from Swami Devi Dyal Institute of Engineering and Technology, Golpura Barwala, Panchkula, Haryana, India.

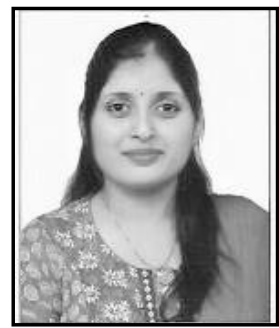

Er. Kiran Gupta is born on 1 April 1985. She completed B. tech (Computer Science Engineering) from Swami Devi Dyal Institute of Engineering and Technology, Golpura, Barwala, Panchkula, Haryana, India in 2007 and M. tech (Computer Science Engineering) from DCSA, Kurukshetra, Haryana, India in 2010. Her areas of interest are Digital Image Processing, Wireless Sensor Networks and Data Mining. Er. Kiran Gupta has 5 years of teaching experience. 